\title{
Role of Dendritic Cells in Chronic HCV Infection and its Correlation with Interferon and Ribavirin Treatment Outcomes
}

\author{
Ahmad Khalaf ${ }^{1}$, Abdallah, A. El-Bially², Mohamed A. Abdeldayem ${ }^{3}$ \\ ${ }^{1}$ Physiology, Zoology Departments, Faculty of Science, Port Said University, Egypt \\ ${ }^{2}$ Microbiology and Immunology Dept., Faculty of Medicine, Zagazig University,Egypt
}

\begin{abstract}
Hepatitis $C$ virus (HCV) infects more than 170 million people worldwide and is a major public health risk. This study was conducted to investigate the role of dendritic Cells in Chronic HCV Infection during the course of Interferon and Ribavirin treatment . The study participants will include three groups of patients. First group include 15 non treated patients with chronic HCV infection as a positive control group. The second group include 15 patients with proven chronic HCV infection and received weekly pegylated Interferon and Ribavirin treatment, This group subdivided into two subgroups: Subgroup A non-responder group with (7) patients. Subgroup B responder group with (8) patients. The third group include 30 healthy patients as a negative control group. Mononuclear cells (PBMCs) were separated from peripheral blood and cultured in presence of either R848 or TNF- $\alpha$ for 48 hrs. At the end of culture period, cytokines, IL-10 and IFN- $\gamma$ were quantified in the culture supernatants by ELISA.
\end{abstract}

Keywords: Chronic HCV Infection, Dendritic Cells (DCs), cytokines, immunotherapy (IT), IL-10, IFN- $\gamma$, PBMCs.

\section{Introduction}

The host immune defense determines successful HCV elimination or chronic infection that often leads to cirrhosis and hepatocellular carcinoma. In particular, Egypt maintains the highest prevalence of infection, affecting and estimated $15 \%$ - $20 \%$ of the population. The origin of the HCV epidemic in Egypt has been attributed to schistosomiasis treatment campaigns in the 1960s and 1970s (Mansour et al., 2010). Furthermore, it is also prospected that the rate of infection will reach its peak between 2010-2020 (Science and Technology for Development fund, 2009).

Dendritic Cells (DCs) are the most potent type of antigen presenting cells and induce immune antiviral response. They are capable of triggering productive immunity and maintaining the state of tolerance to self and non-self antigens (Dolganiuc and Szabo,2011). Human posses two major DC types, myloid DCs (mDCs) and plasmacytoid DCs (pDCs). pDcs produce more interferon- $\alpha$ (IFN- $\alpha$ ) per cell than any other cell type in the body, proinflammatory cytokines, and antigen presentation during viral infection making them aparticularly important component of the antiviral immune response. mDCs are derived from amyloid bone marrow precursor, produce large ammounts of interleukin-12 (IL-12) after bacterial or viral infection and prime Th1 T-cell responses (Liang et aL., 2009). All DCs express high levels of Toll-like receptors (TLRs), which are an important family of pathogen recognition receptors. Ligation of these receptors results in Dc maturation and inflammatory cytokine secretion and is a key event in the initiation of innate immune response (Ryan and Farrelly, 2011).

Currently, the standard therapy is a combination between pegylated interferon and ribavirin. However, the treatment efficiency of this therapy is variable depending on the HCV genotype, it is approximately 50\% effective in treating HCV genotypes 1 and 4 and approximately 80\% effective in treating HCV genotypes 2 and 3. In addition, this combination therapy has severe adverse side effects as well as a high cost (Guo et al.,2012). The mechanisms of HCV persistence remain elusive and are largely related to inefficient clearance of the virus by the host immune system. The key players in immune response to HCV could be dendritic cells (Weigand et al., 2012). During the past decade, multiple research groups were focused in DCs, in hopes of unraveling an HCV-specific DC signature or DC-dependent mechanisms of anti-viral immunity which will lead to a successful HCV elimination strategy (Dolganiuc and Szabo, 2011). Interestingly DCs from HCV carriers show impaired maturation, determined by absence of cell surface markers, as well as reduced IL-12 product loss of DC function is probably a direct consequence of $\mathrm{HCV}$ infection. Intitro generated and maturated DC therefore might be the most potent candidate for a cell-based HCV vaccine (Weigand et al., 2012).

\section{Material and Methods}

This study was conducted at the Immunology Research Laboratory, Microbiology and Immunology Department, Faculty of Medicine, Zagazig University from April 2015 to September 2015. The study participants included three groups of patients. The first group (non treated $\mathrm{HCV}$ patients) included 15 patients, 5 females and 10 males, aged 27 - 58 years (median age 44 years), with chronic HCV infection who were defined on the basis of standard diagnostic criteria positive for both anti-HCV antibody and HCV RNA for more than 6 months, and the serum ALT level greater than the upper limit of normal (40 U/L). Patients were excluded if they had anemia (hemoglobin level $<13 \mathrm{gm} / \mathrm{dL}$ for men and $<12$ $\mathrm{gm} / \mathrm{dL}$ for women), neutropenia (neutrophil count $<1,500$ cells/ mm3), thrombocytopenia (platelet count $<70,000$ cells/ $\mathrm{mm} 3$ ), coinfection with hepatitis B virus or HIV, alcohol or drug abuse, decompensated cirrhosis of the liver (Child-Pugh 


\section{International Journal of Science and Research (IJSR) \\ ISSN (Online): 2319-7064 \\ Index Copernicus Value (2013): 6.14 | Impact Factor (2014): 5.611}

class B or C), autoimmune liver disease, malignancy, organ transplantation or immunosuppressive therapy, pregnancy, poorly controlled autoimmune disease, cardiopulmonary disease, and neuropsychiatric disorders (Liang et al., 2011).

The second group (treated HCV patients) included 15 patients with proven chronic HCV received weekly PEGIFN a-2b $(1.5 \mu \mathrm{g} / \mathrm{kg})$ and daily weight-based ribavirin at a dose of $1,000 \mathrm{mg}$ with body weight $<75 \mathrm{~kg}$ ) or $1,200 \mathrm{mg}$ with body weight $\geq 75 \mathrm{~kg}$ ) for 24 weeks and followed for additional 24 weeks after the cessation of therapy (Liang et al., 2011). They were chosen according to the previous inclusion and exclusion criteria of non-treated patients. They attended El-Asar Hospital Hepatology Unit (Damiatta, Egypt) for pegylated IFN and Ribavirin therapy and was diagnosed by anti-HCV ELISA assay and quantitative HCV PCR. This Group was divided into two subgroups, subgroup A (non-responders) and subgroup B (responders). The subgroup A included seven patients out of the 15 treated patients, 3 females and 4 males aged 18 - 60 years (median age 35years), had failed IFN-based therapy as evidenced by persistent or inadequate reduction in HCV RNA during therapy. They are called non responders. They are now on conservative treatment for liver support. The subgroup B included 8 patients out of the 15 treated patients, 3 males and 5 females, aged 20 - 50 years old (median age 29years), achieved sustained virologic response as evidenced by undetectable serum HCV RNA 6 months after treatment.

The third group included 30 healthy control (non-HCV blood donors), 18 males and 12 females, aged 25 - 53 years old (median age 44years). They were negative for both antiHCV antibody and HCV RNA in serum.

A written informed consent was taken from each individual after explaining the nature of investigation as well as the purpose of the study in accordance with the ethical standards of the responsible regional committee.

\section{Blood Sampling}

Ten $\mathrm{ml}$ of fresh peripheral blood were obtained from the study participants by venous puncture and collected into preservative-free heparin containing tubes (10 U heparin / $\mathrm{ml}$ blood), mixed gently after collection.

\section{Separation of peripheral blood mononuclear cells}

Heparinized blood was diluted 1:1 with phosphate buffer saline (PBS) in $15 \mathrm{ml}$ conical centrifuge tubes (Falcon tubes) and mixed gently by inversion. Diluted blood was completely layered on an identical volume of the density gradient (Lymphoflot; Dieieich, Germany), The tubes were kept at a $45^{\circ}$ angle and the diluted blood was allowed to run down side of tubes without allowing the two solutions to mix. Then, the tubes were transferred to the centrifuge without disturbing the interface. Samples were centrifuged for 30 minutes (min) at $400 \mathrm{xg}$ at room temperature. The PBMCs interface was carefully removed by pipetting and was washed twice with PBS supplemented with $2 \%$ heatinactivated fetal calf serum solution by centrifugation for 10 min at 400xg, so that a visible pellet of PBMCs was formed at the bottom of each tube. All the supernatants that occupied the bottom of the cone of each tube was removed carefully by inversion in order to leave each cell pellet intact. The pellet was re- suspended in $2 \mathrm{ml}$ of RPMI (Roswell Park Memorial Institute) for counting by haemocy- tometer. The cells were tested for their viability by trypan blue exclusion.

\section{Culture medium and in vitro generated monocytes- derived DCs}

Dendritic cells were generated from PBMCs. The cells were cultured at $1 \times 10^{6}$ cells per mL RPMI-1640 containing $10 \%$ fetal calf serum, $2 \%$ penicillin-streptomycin $(\mathrm{P} / \mathrm{S})$ in 6 -well plates with GM-CSF (50 ng $/ \mathrm{mL}$ ). The cells were incubated for two hours at $37^{\circ} \mathrm{C}$ in humidified, 5\% $\mathrm{CO}_{2}$ (Carbon dioxide) atmosphere. After the two hours, the non adherent cells were discarded by inversion of the plate. Fresh medium containing GM-CSF and IL-4 was added and the cells were examined under the inverted microscope at $\times 400$ magnification to detect the adherent monocytes. The cells were fed every 2 days with complete fresh media to generate the immature MoDCs. Maturation was induced by stimulation of immature DCs with either R848 (100 ng/mL) or recombinant TNF- $\alpha(100 \mathrm{ng} / \mathrm{ml})$ to the culture on sixth well for 48 hrs. Lipopolysaccharide from Salmonella minnesota, at a concentration of $10 \mathrm{ng} / \mathrm{ml}$ was used as a positive control for maturation of immature MoDCs. Culture supernatants were harvested, collected and stored at $-20^{\circ} \mathrm{C}$ till use for cytokines measurement (IL-10 and IFN- $\gamma$ ) avoiding repeated freezing and thawing (Liang et al., 2011).

\section{Identification of monocytes - derived DCs:}

Briefly, the adherent DCs were harvested by a scraper. Aliquots of the cells to be analyzed were incubated in PBS containing $2 \%$ fetal calf serum. The cell suspension was then incubated with a predetermined optimal concentration of the appropriate fluorescently labeled mAbs against human cell surface molecules on ice for $30 \mathrm{~min}$. After washing with fluorescence activated cell sorting [FACS] buffer, cells were fixed in $1 \%$ paraformaldehyde (PFA)-containing FACS buffer. The cells were analyzed by standard flow cytometry using a FACS-Calibur assisted by Cell Quest software (BD Pharmingen) (Zhang et al., 2008). There was an increase in the expression of HLA-DR and CD83 and decrease in the expression of CD14 on DCs compared to the freshly isolated monocytes.

\section{Cytokines Measurement}

The levels of IL-10 and IFN- $\gamma$ in the culture supernatants were determined by sandwich enzyme-linked immunosorbent assay (ELISA).

\section{Quantitative determination of IL-10:}

Results were calculated against standard curve. The standard curve is used to determine the amount of IL-10 in an unknown sample. The standard curve is generated by plotting the O.D. obtained for each of the standard concentrations on the vertical (Y) axis versus the corresponding IL-10 concentration $(\mathrm{pg} / \mathrm{ml})$ on the horizontal $(\mathrm{X})$ axis.

\section{Quantitative determination of IFN- $\gamma$}

Results were calculated against standard curve. The standard curve is used to determine the amount of IFN- $\gamma$ in an unknown sample. The standard curve is generated by plotting the O.D. obtained for each of the standard concentrations on the vertical (Y) axis versus the corresponding IFN- $\gamma$. 


\section{International Journal of Science and Research (IJSR) \\ ISSN (Online): 2319-7064 \\ Index Copernicus Value (2013): 6.14 | Impact Factor (2014): 5.611}

concentration (IU/ml) on the horizontal (X) axis.

\section{Statistical Analysis}

All analyses were performed using Statistical Package for the Social Sciences software (SPSS version 21.0). Data were expressed as mean and median values \pm 1 standard deviation (SD). Data were entered as variables. All analyses were performed using parametric tests. For comparisons between groups within the same variable, one-way ANOVA (was used. The correlation coefficient $r$ was generated using the Pearson correlation. All tests were 2tailed and P (probability) values of 0.05 or less were considered to be statistically significant.

\section{Results}

\section{Morphological changes of MoDCs}

After 6 days of cultivation, immature MoDCs were generated. Morphology was determined by inverted microscope at $400 \mathrm{X}$ power. They were bigger in size, irregular in shape, had pseudopodia-like projections and were adherent to the culture plate. Mature MoDCs were generated after addition of either R848 $(100 \mathrm{ng} / \mathrm{ml})$ or recombinant TNF $(100 \mathrm{ng} / \mathrm{ml})$ to the culture medium on the sixth day of cultivation for 48 hours. They were also examined under the inverted microscope to determine their morphology. They appeared bigger in size, had more pseudopodia-like projections and were strongly adherent to the plate.

\section{Phenotypical changes of MoDCs}

There was an increase in the expression of HLA-DR and CD83 and decrease in the expression of CD14 on DCs compared to the freshly isolated monocytes. There was an increase in the forward side scatter intenisty compared with their monocyte precursors as well.

\section{Functional changes of MoDCs}

The supernatant of the culture medium was collected to determine the level of cytokines produced by DCs.

\section{LPS induced maturation}

For production of matured and functioning DCs from monocytes, LPS (10 $\mathrm{ng} / \mathrm{ml})$ was used as a known DCs activation stimulant (positive control). Only 3 cases from each group (healthy controls, non-treated HCV patients, responders and non-responders to PEG-IFN and RBV therapy) were selected for LPS induced maturation.

\section{- LPS induced IL-10 production:}

After maturation of MoDCs by LPS, IL-10 was measured, There were no significant differences in mean values of IL10 production among all study groups, $(p<0.05)$. However, there was a trend of increasing IL-10 production in the 3 healthy controls and the 3 responders as compared to the 3 non-treated HCV patients and the 3 non-responders. The mean values of IL-10 production in all culture media including the negative control, after LPS and after R848 induced maturation of the 3 healthy controls were (31.76 \pm 3.95), (243.5 \pm 11.3$)$ and $(237 \pm 12)$ respectively. On the other hand, those of the 3 non-treated HCV patients were (25.56 \pm 11.36), $(230.9 \pm 21.6)$ and $(211.7 \pm 29.3)$ respectively (Fig.1). The mean values of IL-10 production in all culture media including the negative control, after LPS and after R848 induced maturation of the 3 responders were (32 \pm 12.76), $(246 \pm 4.5)$ and $(202.2 \pm 8)$ respectively. Meanwhile, those of the 3 non-responders were (19.7 \pm 9.25$)$, (222.8 \pm 10.9$)$ and (195 \pm 8.7) respectively (Fig.2). From the previous results, it was noticed that the mean value of IL-10 production after LPS induced maturation was higher than that produced after R848 induced maturation.

\section{- IFN- $\gamma$ production after LPS induced maturation}

After maturation of MoDCs by LPS and IFN- $\gamma$ was measured. There were significant differences in mean values among all groups of patients, $(\mathrm{P}>0.05)$. However, there was a trend of increasing IFN- $\gamma$ production in the 3 non-treated HCV patients and 3 responders as compared with the 3 healthy controls and the 3 non-responders. The mean values of IFN- $\gamma$ production in all culture media including the negative control, after LPS and after R848 induced maturation of the 3 nontreated HCV patients were $(0.15 \pm 0.13),(1.56 \pm 0.09)$ and $(1.23 \pm 0.2)$ respectively. Meanwhile, those of the 3 healthy controls were $(0.2 \pm 0.07)$, $(1.5 \pm 0.2)$ and $(1.2 \pm 0.3)$ respectively (Error! Reference source not found.3). The mean values of IFN- $\gamma$ production in all culture media including the negative control, after LPS and after R848 induced maturation of the 3 responders were $(0.096 \pm 0.01)$, $(0.94 \pm 0.3)$ and $(0.77 \pm 0.1)$ respectively. Meanwhile, those of the 3 non-responders were $(0.09 \pm 0.01),(0.7 \pm 0.1)$ and $(0.54 \pm 0.1)$ respectively (Error! Reference source not found.4).

\section{IL-10 cytokine analysis:}

\section{- TNF induced $I L-10$ production}

There were high significant differences in mean values of IL10 among all study groups, ( $>0.001)$. The mean values of IL10 production after recombinant TNF $(100 \mathrm{ng} / \mathrm{ml})$ induced maturation were higher among healthy controls (238.25 \pm $12.57)$ and responders (241.2 \pm 9.02$)$ when compared to nontreated HCV patients $(128.87 \pm 83.5)$ and non-responders $(224.17 \pm 4.28)$ respectively (Error! Reference source not found.5).

\section{- $R 848$ induced IL-10 production}

There were high significant differences in mean values of IL10 among all study groups, ( $>0.001)$. The mean values of IL10 production after $\mathrm{R} 848(100 \mathrm{ng} / \mathrm{ml})$ induced maturation were higher among healthy controls $(232.36 \pm 15.37)$ and responders $(217.6 \pm 22.88)$ when compared to non-treated HCV patients $(121.6 \pm 90.3)$ and non-responders (215.14 \pm 24.66) respectively (Error! Reference source not found.5).

\section{IFN- $\gamma$ cytokine analysis:}

\section{- TNF induced IFN- $\gamma$ production:}

There were significant differences in mean values of IFN- $\gamma$ among all study groups, $(\mathrm{p}>0.05)$. The mean values of IFN- $\gamma$ production after induction of maturation by recombinant TNF were higher among non-treated HCV patients $(1.5 \pm 0.54)$ and responders $(1.2 \pm 0.19)$ when compared to healthy controls $(1.3 \pm 0.188)$ and non-responders $(0.84 \pm 0.11)$ respectively (Error! Reference source not found.6).

\section{- R848 induced IFN- $\gamma$ production:}

There were significant differences in mean values of IFN- $\gamma$ among all study groups, (p>0.05). The mean values of IFN- $\gamma$ production after induction of maturation by R848 were higher 


\section{International Journal of Science and Research (IJSR) \\ ISSN (Online): 2319-7064 \\ Index Copernicus Value (2013): 6.14 | Impact Factor (2014): 5.611}

among non-treated HCV patients (1.4 \pm 0.51) and responders $(0.88 \pm 0.1)$ when compared to healthy controls $(1.2 \pm 0.2)$ and non-responders $(0.7 \pm 0.08)$ respectively (Error! Reference source not found.6).

- Correlation between IL-10 and IFN- $\gamma$ production after $\mathbf{R 8 4 8}$ induced maturation:

There was non significant inverse correlation between IL10 and IFN- $\gamma$ production after MoDCs induced maturation by R848 $(100 \mathrm{ng} / \mathrm{ml})$ in non-treated $\mathrm{HCV}$ patients $(\mathrm{r}=$ 0.296, $\mathrm{p}<0.05$ ) (Error! Reference source not found.7). and non-responders $(r=-0.387, \mathrm{p}<0.05)$ (Error! Reference source not found.8). Meanwhile, there was non significant positive correlation between IL-10 and IFN- $\gamma$ production after MoDCs induced maturation by R848 $(100 \mathrm{ng} / \mathrm{ml})$ in responders $(r=0.344, \mathrm{p}<0.05)$ (Error! Reference source not found.9).

\section{Discussion}

Dendritic cell activity is critical for the generation of a robust polyclonal T-cell response. Ryan and his collegues (2011) explored the hypothesis that the failure of an individual to mount an effective T-cell response during acute HCV infection is because of viral impairment of DC function. Despite extensive studies, there is little overall consensus on this topic. Reduced numbers of circulating DCs, reduced levels of co-stimulatory molecule expression and failure to stimulate allogeneic mixed lymphocyte reactions (MLRs) have been reported by some investigators (Della Bella et al., 2007) but not others (Piccioli et al., 2005). Similarly, impaired responses to TLR agonists by DCs from patients with HCV have been reported (Miyazaki et al., 2008), while others have demonstrated their ability to induce robust responses (Echeverria et al., 2008).

In the present study MoDCs generated invitro from PBMCs of healthy controls, non-treated HCV patients, responders and non-responders to therapy. This method because the low frequency of DCs in the circulation and the limited volume of blood available from patients precluded us from assessing the functional properties of these cells directly. To overcome this obstacle, many groups have studied the phenotype and function of MoDCs obtained from patients with chronic HCV infection. MoDCs are cells with myeloid DC phenotype, obtained in relatively high numbers by culturing CD14+ monocytes with GM-CSF and IL-4 for 57 day (Michielsen et al., 2010). We preferred myeloid DCs as they are higher in number with longer life span than plasmacytoid DCs. Moreover pDCs are difficult to be generated invitro. MoDCs have potent antigen presentation capacity and are the cell types most likely to be used in a DC vaccine. However, plasmacytoid is still important as they have better ability of viral killing.

Potent TLR7, 8 Ligand which is R848, or resiquimod, used a small guanosine analog molecule for induction of maturation of MoDCs. It is known to signal through either TLR7 or TLR8, which mimics natural ligands, fragments of viral ssRNA, in a manner yet unclear. R848 is known to induce IFN- $\alpha$ from PDCs, but it also induces high levels of inflammatory cytokines such as IL-6, TNF- $\alpha$, IL-12, and IL-10 from monocytes and myeloid dendritic cells (Marshall et al., 2007). R848 seems to be a good candidate for induction of differentiation to a DC phenotype with ability to induce naive CD4+ $\mathrm{T}$ cells towards the Th17 lineage (Jensen and Gad 2010). IFN- $\alpha$ is the mainstay treatment for chronic hepatitis $\mathrm{C}$ virus infection but with partial success. So that, activation of TLR7 and/or TLR8 represents a powerful and novel therapeutic strategy for the treatment of various viral infections.

In the present study, The production of two cytokines that have received greater attention for their potential to regulate Th2 and Th1 response, IL-10 and IFN- $\gamma$ respectively, in the supernatants of 7 days cultures of PBMCs isolated from healthy controls, non-treated HCV patients, responders and non-responders to therapy and assessed the in-vitro capacity of TNF- $\alpha$ and R848 as stimulators for maturation of DCs.

IL-10 is a pleiotropic cytokine with important immunoregulatory functions in which influence activities of many of the cell-types in the immune system. The main biological function of IL-10 appears to be exerted on DCs and macrophages. It is a potent inhibitor of antigen presentation. IL-10 inhibits the differentiation of DCs from monocyte precursors, and it also inhibits DC maturation. Thus, many of the immuno-inhibitory characteristics of IL-10 can be traced to their effect on APCs to prevent the production of the Th1associated cytokines IL-2 and IFN- $\gamma$, and also the Th2associated IL-4 and IL-5 (Mosser and Zhang 2008). IL-10 was originally described as a cytokine synthesis inhibitory factor, but recent studies have demonstrated that IL-10 produced by Th17 cells restrains the pathologic effects of Th17 .Furthermore, there is strong evidence of a substantial genetic component to IL-10 production; the $-1082 \mathrm{G} / \mathrm{G}$ genotype is known to be related to increased IL 10 production and is associated with a high risk of inefficient HCV clearance and resistance to IFN treatment (Yoneda et al., 2011). It is suggested that a Th1-type rather than a Th2-type response may be beneficial for controlling and clearing HCV.

The present study showed high significant differences in mean values of IL-10 production by MoDCs among all study groups after recombinant TNF and R848 induced maturation. It was higher among healthy controls and responders when compared to non-treated HCV patients and non-responders respectively.These data are consistent with that reported by Barnes and his collegues (2008). These results may be accounted for by patient cohorts with different amount of liver inflammation / fibrosis and that the alteration in DC function described is induced in part by liver pathology rather than by HCV. Meanwhile, (Ryan et al., 2011) reported higher production of IL-10 in non- treated HCV patients than healthy controls and (Liang et al., 2011) reported that as well beside higher IL-10 in non-responders than responders. Rehermann and Nascimbeni (2005) reported that patients who develop a chronic infection show a predominant Th2 response that down-regulates the Th1 response. Wan and his collegues (2009) showed that Th2 cytokines (IL-4, IL-5, and IL-10) were markedly increased in non SVR patients. The overall increases in Th2, which down-regulated the cell-mediated immune response may have led to viral resistance, even though high amounts of Th1 cytokine were expressed in serum.

Although IFN- $\gamma$ has some antiviral activity, it is much less 


\section{International Journal of Science and Research (IJSR) \\ ISSN (Online): 2319-7064 \\ Index Copernicus Value (2013): 6.14 | Impact Factor (2014): 5.611}

active in this regard than type I IFNs. IFN- $\gamma$ is involved in the regulation of nearly all phases of the immune and inflammatory responses, including the activation and differentiation of T cells, B cells, NK cells, macrophages, and others. It is therefore best regarded as a distint immunoregulatory cytokine. IFN- $\gamma$ secretion is a hallmark of Th1 lymphocytes. It is also secreted by nearly all CD8 T cells, by some Th0 cells, and by NK cells (Gattoni et al., 2006).

IFN- $\gamma$ production by professional APCs [monocyte/macrophage, dendritic cells] acting locally may be important in cell self-activation and activation of nearby cells. IFN- $\gamma$ secretion by NK cells and possibly professional APCs is likely to be important in early host defense against infection, whereas $\mathrm{T}$ lymphocytes become the major source of IFN- $\gamma$ in the adaptive immune response (Schroder et al., 2004).

In the present study, it was found a significant differences in mean values of IFN- $\gamma$ production by MoDCs among all study groups. The mean values of IFN- $\gamma$ production after stimulation by recombinant TNF and R848 were higher among non-treated HCV patients and responders when compared to healthy controls and non-responders respectively. These findings are in agreement with that reported by Liang and his colleagues (2011) as regard responders and non-responders, but they observed that IFN$\gamma$ production showed no significant difference between healthy controls and non-treated HCV patients. This controversy may be attributed to different genotypes of HCV. Moreover, they screened the same patients before and after treatment, but we chose different groups of patients. However, reduced IFN- $\gamma$ production by PBMCs after stimulation with ligands for TLR had been reported in chronic hepatitis $\mathrm{C}$ patients receiving therapy regardless of treatment outcome (Hammond et al., 2010).

The current results directed us to further analyze and study the correlation between IL-10 and IFN- $\gamma$. The results analysis showed no significant inverse correlation between IL-10 and IFN- $\gamma$ production after MoDCs induced maturation by R848 in non-treated HCV patients and nonresponders as well. Meanwhile, there was no significant positive correlation between IL-10 and IFN- $\gamma$ production after MoDCs induced maturation by R848 in responders.

The results demonstrated that the level of IFN- $\gamma$ and IL-10 were significantly higher in responders than nonresponders. These results were consistent with that reported by (Liang et al., 2011) and (Ryan et al., 2011) as regard IFN- $\gamma$ and (Barnes et al., 2008) as regard IL-10. On the other hand these results were different from that reported by (Barnes et al., 2008) as regard IFN- $\gamma$ and (Liang et al., 2011), (Ryan et al., 2011) as regard IL-10.

\section{References}

[1] Barnes, E., M. Salio, V. Cerundolo (2008). "Monocyte derived dendritic cells retain their functional capacity in patients following infection with hepatitis C virus." J Viral Hepat 15(3): 219-28.
[2] Della Bella, S., A. Crosignani, A. Riva (2007). "Decrease and dysfunction of dendritic cells correlate with impaired hepatitis C virus-specific CD4+ T-cell proliferation in patients with hepatitis $\mathrm{C}$ virus infection." Immunology 121(2): 283-92.

[3] Dolganiuc, A. and G. Szabo (2011)" .)Dendritic cells in hepatitis C infection: can they (help) win the battle?" J Gastroenterol 46(4): 432-47.

[4] Echeverria, I., A. Zabaleta, L. Silva (2008). "Monocyte-derived dendritic cells from HCV-infected patients transduced with an adenovirus expressing NS3 are functional when stimulated with the TLR3 ligand poly(I:C)." J Viral Hepat 15(11): 782-9.

[5] Gattoni, A., A. Parlato, B. Vangieri (2006). "Interferongamma: biologic functions and HCV therapy (type I/II) (1 of 2 parts)." Clin Ter 157(4): 377-86.

[6] Guo, Z., H. Zhang, H. Rao (2012). "DCs pulsed with novel HLA-A2-restricted CTL epitopes against hepatitis $\mathrm{C}$ virus induced a broadly reactive anti-HCV-specific $\mathrm{T}$ lymphocyte response." PLoS One 7(6): e38390.

[7] Hammond, T., S. Lee, M. W. Watson (2010). "Decreased IFNgamma production correlates with diminished production of cytokines by dendritic cells in patients infected with hepatitis $\mathrm{C}$ virus and receiving therapy." J Viral Hepat 18(7): 482-92.

[8] Jensen, S. S .and M. Gad (2010). "Differential induction of inflammatory cytokines by dendritic cells treated with novel TLR-agonist and cytokine based cocktails: targeting dendritic cells in autoimmunity." J Inflamm (Lond) 7: 37 .

[9] Liang, H., R. S. Russell, N. L. Yonkers (2009). "Differential effects of hepatitis C virus JFH1 on human myeloid and plasmacytoid dendritic cells." J Virol 83(11): 5693-707.

[10] Liang, C. C., C. H. Liu, Y. L. Lin (2011). "Functional impairment of dendritic cells in patients infected with hepatitis $\mathrm{C}$ virus genotype 1 who failed peginterferon plus ribavirin therapy." J Med Virol 83(7): 1212-20.

[11] Marshall, J. D., D. S. Heeke ,M. L. Gesner (2007). "Negative regulation of TLR9-mediated IFN-alpha induction by a small-molecule, synthetic TLR7 ligand." J Leukoc Biol 82(3): 497-508.

[12] Michielsen, A.J., O'Sullivan, J.N.and Ryan, E.J. (2010). Recent advances in the development of novel therapeutics targeting dendritic cells. Recent Pat Endocrine Metabolic Immune Drug Discov 2010; BSP/EMI/E-Pub/00004.

[13] Mosser, D. M. and X. Zhang (2008). "Interleukin-10: new perspectives on an old cytokine." Immunol Rev 226: 205-18.

[14] Piccioli, D., S. Tavarini, S. Nuti (2005). "Comparable functions of plasmacytoid and monocyte-derived dendritic cells in chronic hepatitis $\mathrm{C}$ patients and healthy donors." J Hepatol 42(1): 61-7.

[15] Ryan, E. J. and C. O'Farrelly (2011). "The affect of chronic hepatitis $\mathrm{C}$ infection on dendritic cell function: a summary of the experimental evidence." J Viral Hepat 18(9): 601-7.

[16] Ryan, E. J., N. J. Stevenson, J. E. Hegarty (2011). "Chronic hepatitis C infection blocks the ability of dendritic cells to secrete IFN-alpha and stimulate T-cell proliferation." J Viral Hepat 18(12): 840-51. 


\section{International Journal of Science and Research (IJSR) \\ ISSN (Online): 2319-7064 \\ Index Copernicus Value (2013): 6.14 | Impact Factor (2014): 5.611}

[17] Schroder, K., P. J. Hertzog, T. Ravasi and D. A. Hume (2004). "Interferon-gamma: an overview of signals, mechanisms and functions." J Leukoc Biol75(2): 89-163.

[18] Science and Technology for Development Fund (2009).

[19] Weigand, K., F. Voigt, J. Encke (2012). "Vaccination with dendritic cells pulsed with hepatitis C pseudo particles induces specific immune responses in mice." World J Gastroenterol 18(8): 785-93.

[20] Yoneda, S., T. Umemura, Y. Katsuyama (2011). "Association of Serum Cytokine Levels With Treatment Response to Pegylated Interferon and Ribavirin Therapy in Genotype 1 Chronic Hepatitis C Patients". The Journal of Infectious Diseases 2011;203:1087-95.

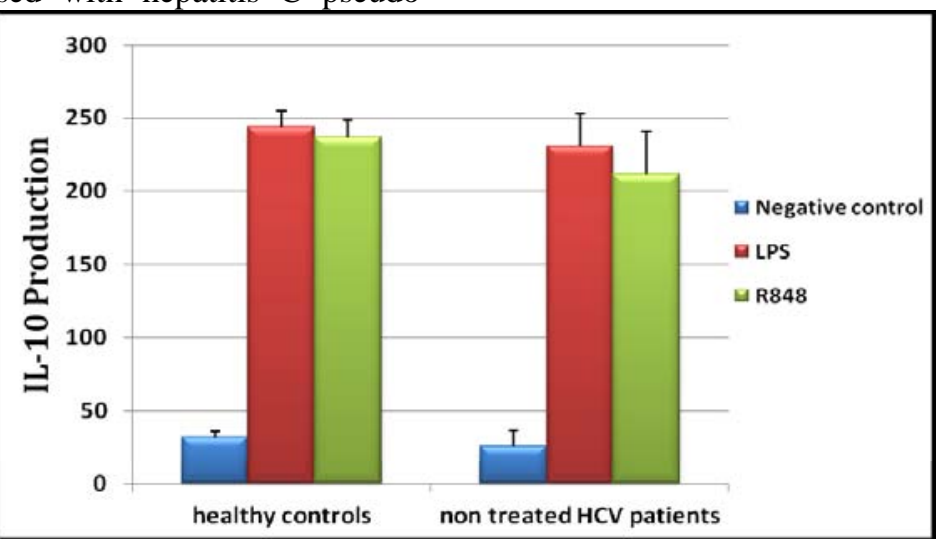

Figure 1: IL-10 production after maturation of MoDCs by adding LPS (10 ng/ml)to the culture medium on the $6^{\text {th }}$ day. IL-10 production is higher with LPS induced maturation (red bars) than R848 induced maturation (green bars). The data are representative of 3 healthy controls and 3 non-treated HCV patients. IL-10 is expressed as mean value $\pm 1 S D$

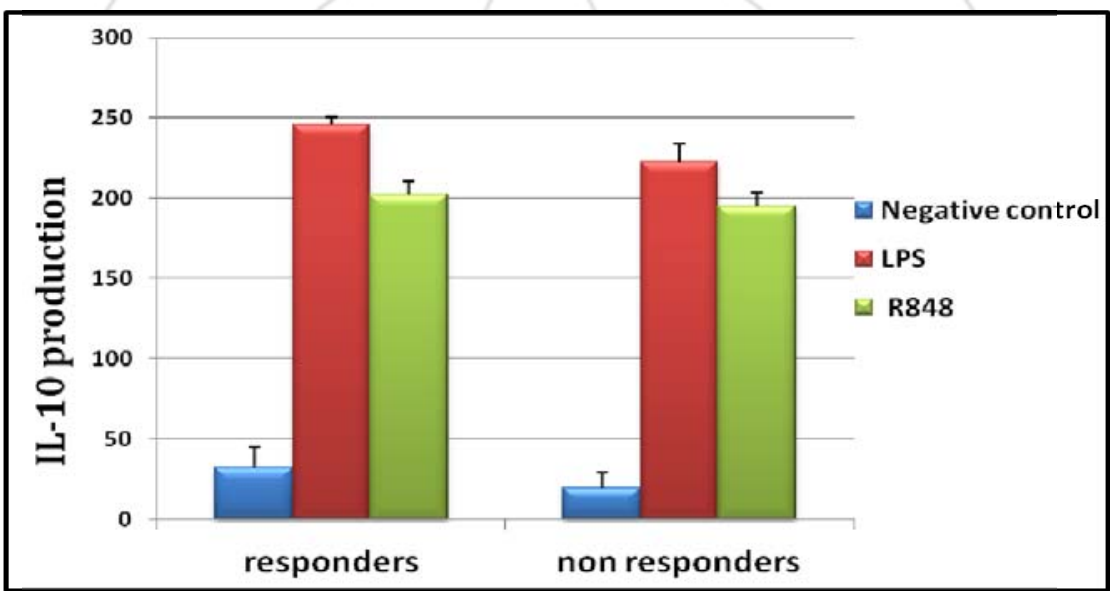

Figure 2: IL-10 production after maturation of MoDCs by adding LPS (10 $\mathrm{ng} / \mathrm{ml})$ to the culture medium on the $6^{\text {th }}$ day. IL-10 production is higher with LPS induced maturation (red bars) than R848 induced maturation (green bars). The data are representative of 3 responders and 3 non-responders. IL-10 is expressed as mean value \pm 1 SD.

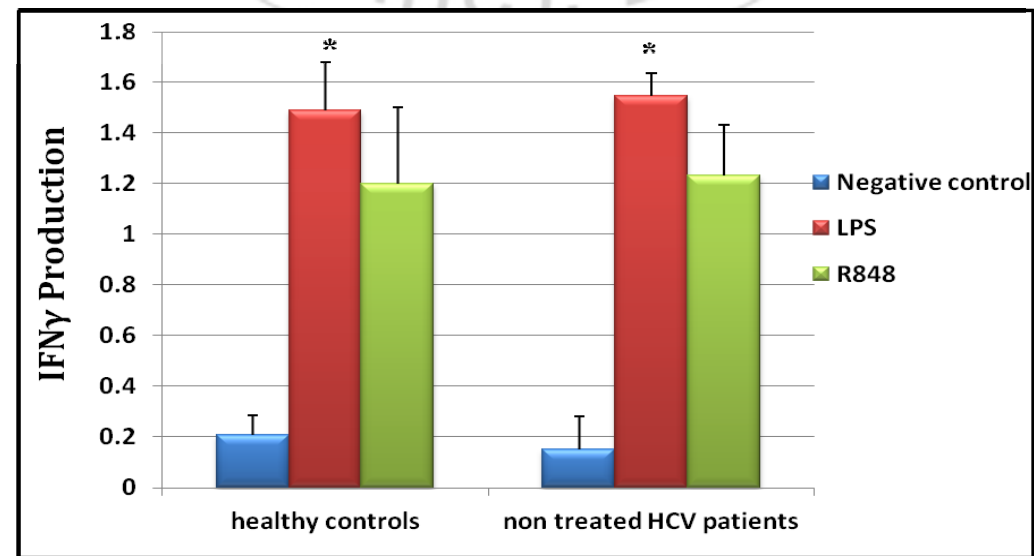

Figure 3: IFN- $\gamma$ production after maturation of MoDCs by adding LPS $(10 \mathrm{ng} / \mathrm{ml})$ to the culture medium on the $6^{\text {th }}$ day. IFN- $\gamma$ production is higher with LPS induced maturation (red bars) than R848 induced maturation (green bars). The data are representative of 3 healthy controls and 3 non-treated HCV patients. IFN $-\gamma$ is expressed as mean value \pm 1 SD, $\mathrm{p}^{*}>0.05$. 


\section{International Journal of Science and Research (IJSR) \\ ISSN (Online): 2319-7064}

Index Copernicus Value (2013): 6.14 | Impact Factor (2014): 5.611

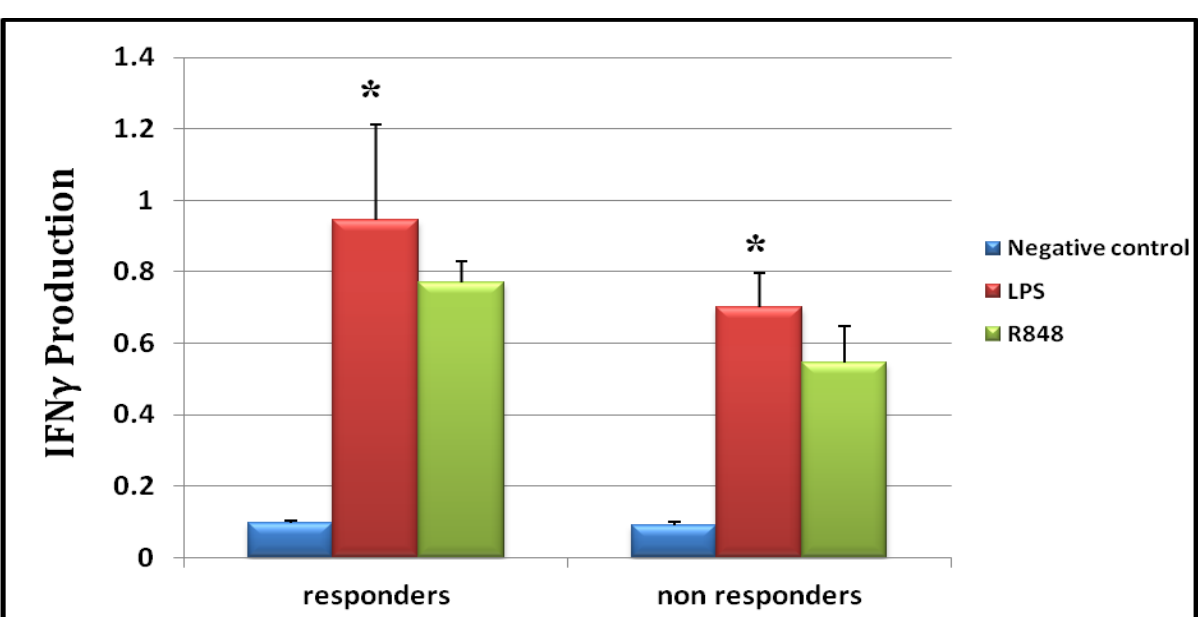

Figure 4: IFN- $\gamma$ production after maturation of MoDCs by adding LPS $(10 \mathrm{ng} / \mathrm{ml})$ to the culture medium on the $6^{\text {th }}$ day. IFN- $\gamma$ production is higher with LPS induced maturation (red bars) than R848 induced maturation (green bars). The data are representative of 3 responders and 3 non-responders. IFN $-\gamma$ is expressed as mean value $\pm 1 \mathrm{SD}, \mathrm{p}^{*>0} 0.05$.

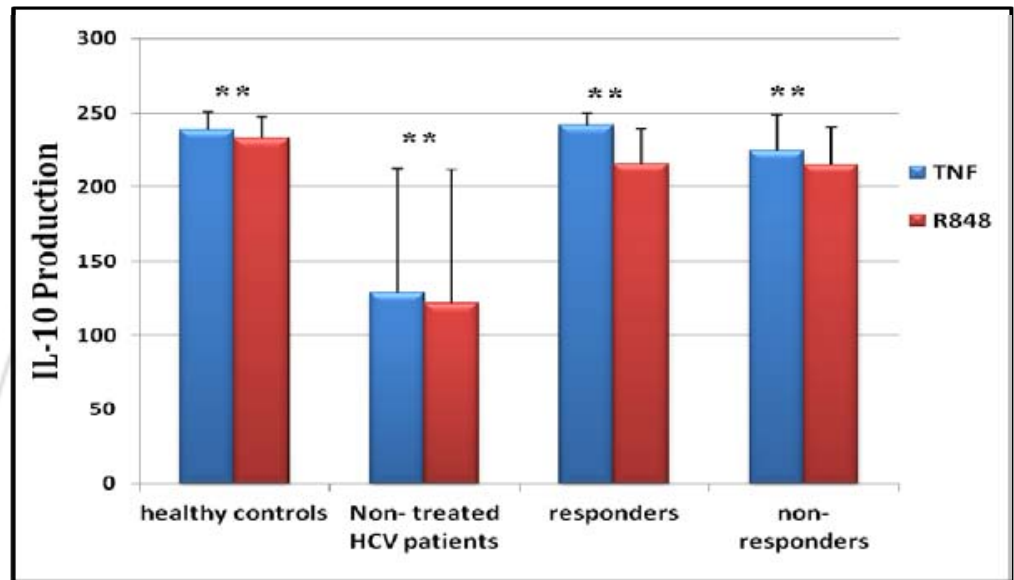

Figure 5: IL-10 production after maturation of MoDCs by adding either TNF $(100 \mathrm{ng} / \mathrm{ml})$ or R848 $(100 \mathrm{ng} / \mathrm{ml})$ to the culture medium on the $6^{\text {th }}$ day. IL-10 production is higher with TNF induced maturation (blue bars) than R848 induced maturation (red bars). The data are representative of 30 healthy controls, 15 non-treated HCV patients, 8 responders and 7 nonresponders. IL-10 is expressed as mean value $\pm 1 \mathrm{SD}, \mathrm{p}^{* *}>0.001$.

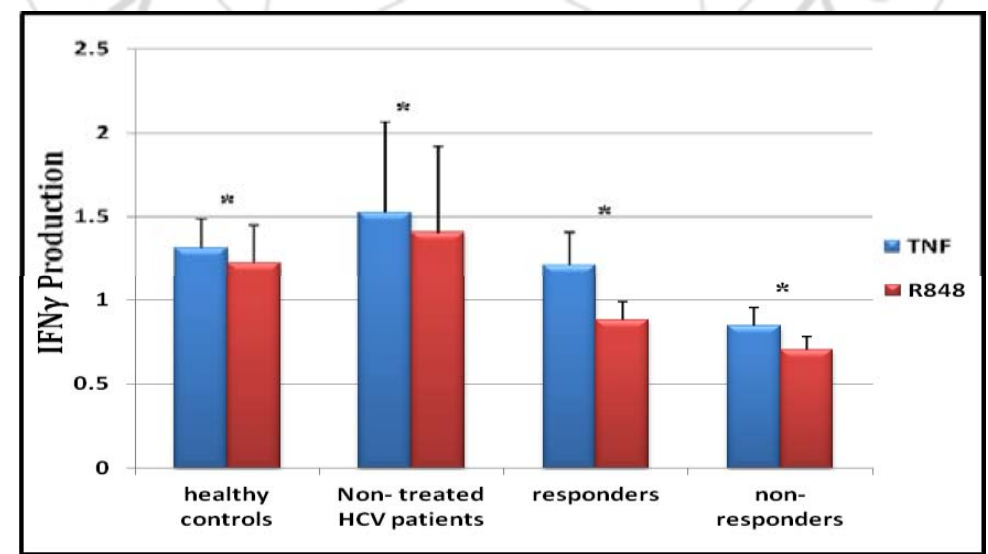

Figure 6: IFN- $\gamma$ production after maturation of MoDCs by adding either TNF $(100 \mathrm{ng} / \mathrm{ml})$ or R848 $(100 \mathrm{ng} / \mathrm{ml})$ to the culture medium on the $6^{\text {th }}$ day. IFN- $\gamma$ production is higher with TNF induced maturation (blue bars) than R848 induced maturation (red bars). The data are representative of 30 healthy controls, 15 non-treated HCV patients, 8 responders and 7 nonresponders. IFN- $\gamma$ is expressed as mean value $\pm 1 \mathrm{SD}, \mathrm{p}^{*}>0.05$. 
International Journal of Science and Research (IJSR)

ISSN (Online): 2319-7064

Index Copernicus Value (2013): 6.14 | Impact Factor (2014): 5.611

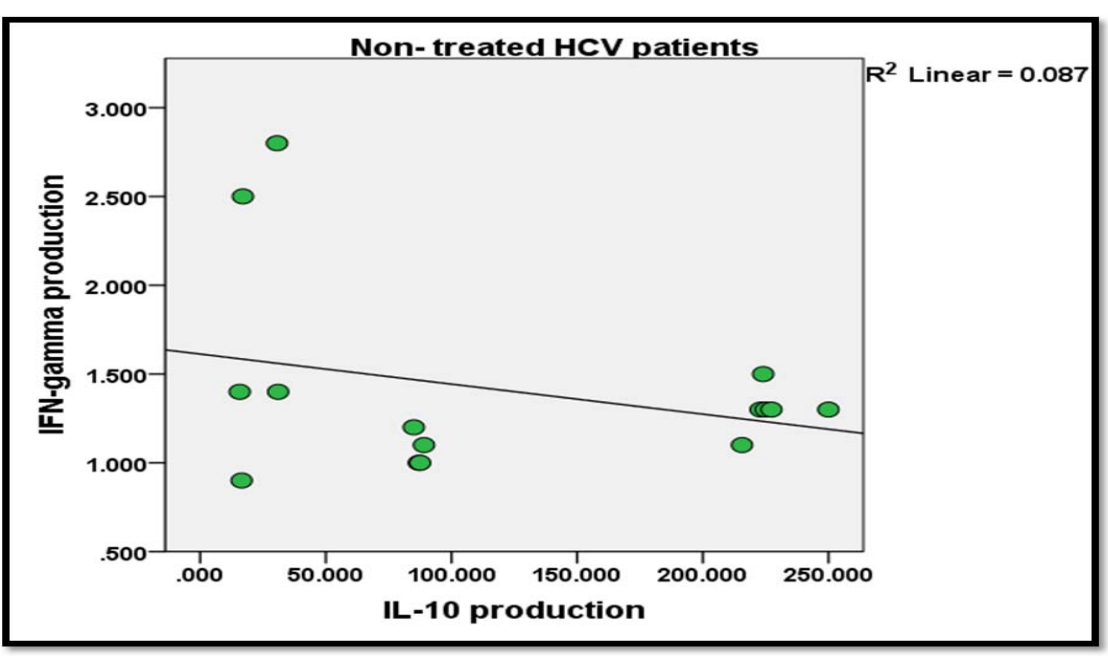

Figure 7: Inverse correlation between IL-10 and IFN- $\gamma$ production after MoDCs induced maturation by R848 in non-treated HCV patients. IL-10 is expressed as mean value on $\mathrm{X}$ axis while IFN- $\gamma$ is expressed as mean value on $\mathrm{Y}$ axis.

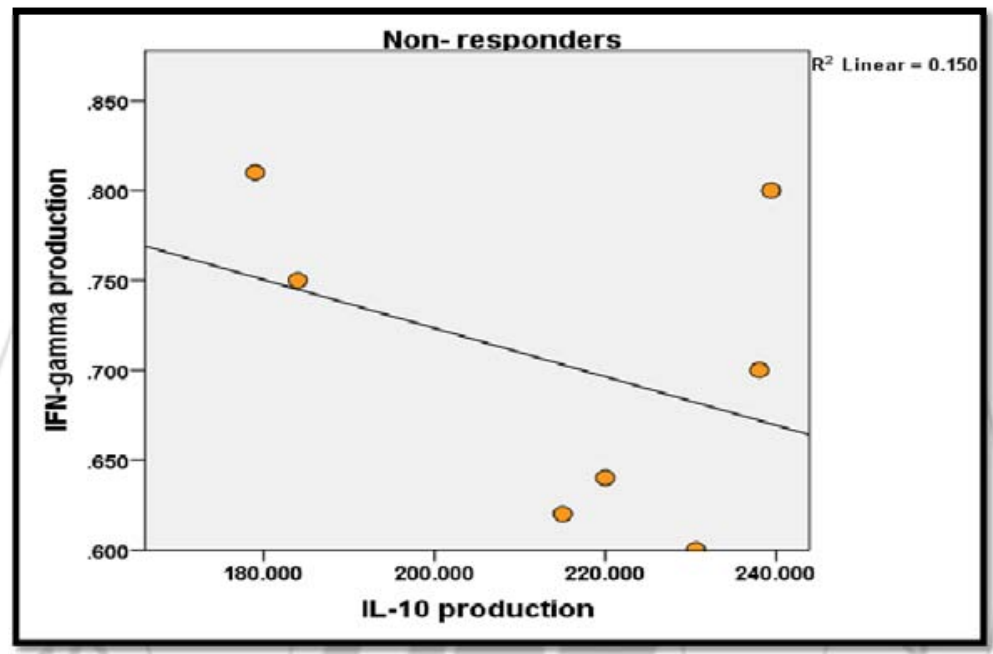

Figure 8: Inverse correlation between IL-10 and IFN- $\gamma$ production after MoDCs induced maturation by R848 in nonresponders. IL-10 is expressed as mean value on $\mathrm{X}$ axis while IFN- $\gamma$ is expressed as mean value on $\mathrm{Y}$ axis.

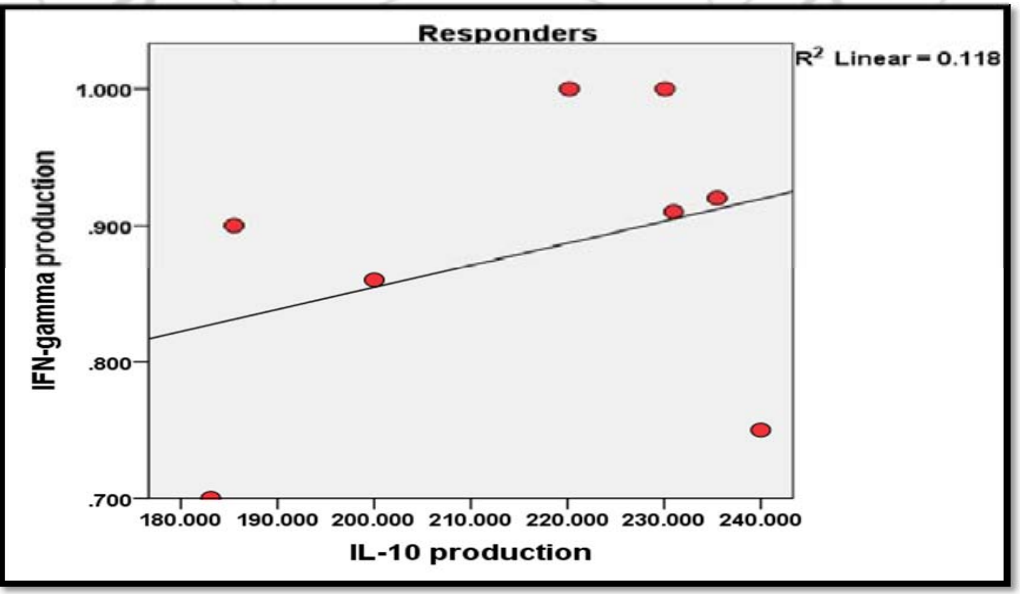

Figure 9: Positive correlation between IL-10 and IFN- $\gamma$ production after MoDCs induced maturation by R848 in responders. IL-10 is expressed as mean value on $\mathrm{X}$ axis while IFN- $\gamma$ is expressed as mean value on $\mathrm{Y}$ axis. 